\title{
THE HATCHER-WALDHAUSEN MAP IS A SPECTRUM MAP
}

\author{
JOHN ROGNES
}

\begin{abstract}
We prove that the map $G / O \rightarrow \Omega W h^{D i f f}(*)$ which was constructed by Waldhausen and proved to be a rational equivalence by Bökstedt, is in fact an infinite loop map if we use a multiplicative infinite loop space structure on the target. As an application we investigate the obstruction to improving Bökstedt's two-primary results on splitting the étale $K$-theory space $J K(\mathbb{Z})$ off from $K(\mathbb{Z})$, to the unlooped space or spectrum level.
\end{abstract}

\section{INTRODUCTION}

In this paper we give a manifold model for Waldhausen's $A$-theory space $A(*)$, geared towards studying the multiplicative spectrum structure induced by smash products of spaces on its one-component. This allows us to infinitely deloop the rational equivalence considered in [Wa $\mathrm{M}]$ and [Bö]

$$
G / O \rightarrow \Omega W h^{D i f f}(*)
$$

to a spectrum map, when $W h^{D i f f}(*)$ is made into a spectrum using this multiplicative structure (Corollary 1.4).

As an application we consider delooping Bökstedt's two-complete splitting of the loop space of his model for "étale $K$-theory of the integers," $\Omega J K(\mathbb{Z})[\mathrm{Bö}]$, off from the loop space of algebraic $K$-theory of the integers, $\Omega K(\mathbb{Z})$, to the unlooped space or spectrum level.

Let $S G=Q\left(S^{0}\right)_{1}$ and $K(\mathbb{Z})_{1}$ denote the multiplicative (smash- and tensor product) spectra. We prove that if the map $S G \rightarrow K(\mathbb{Z})_{1}$ factors through the image of $J$ spectrum (over $e: S G \rightarrow J_{\otimes}$ ), then indeed $J K(\mathbb{Z})_{1}$ splits off $K(\mathbb{Z})_{1}$ as two-completed spectra (Theorem 2.1).

Let us note that the argument provides a fibration of spectra

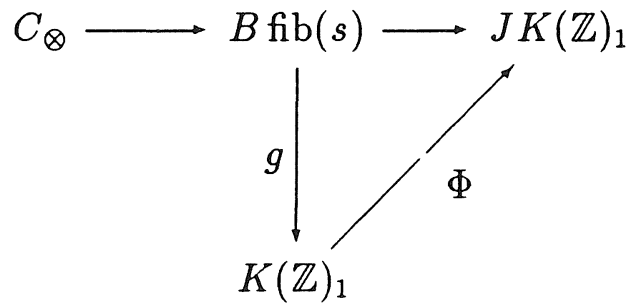

where the projection map factors through $K(\mathbb{Z})_{1}$, but we need to assume a null homotopy of the composite $C_{\otimes} \rightarrow K(\mathbb{Z})_{1}$ in order to obtain the splitting result. 
Our methods are explicitly geometric in $\S 1$, and more formal in $\S 2$ and $\S 3$, relying on the theorems of Madsen, Snaith and Tornehave [MST] in various guises, in particular as presented by Adams [Ad]. See Proposition 2.2 and Corollary 2.3.

The author thanks Marcel Bökstedt for proposing the problem of delooping the map $G / O \rightarrow \Omega W h^{D i f f}(*)$, and for numerous extremely helpful and friendly discussions. Further thanks to Jørgen Tornehave and Ib Madsen for explanations, and for making possible a delightfully inspiring year's stay at Århus University. Thanks also to John Klein for references to Ganea's work, and to Paul Taylor for his $\mathrm{TEX}_{\mathrm{E}}$ package.

\section{§1. Multiplicative Partitions}

In this section we will construct the map $G / O \rightarrow \Omega W h^{D i f f}(*)$ as a spectrum map, as the map of homotopy fibers of the horizontal maps in a homotopy commutative diagram of spectra :

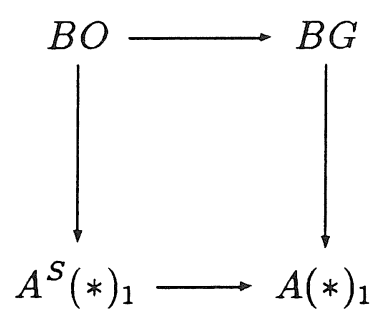

Here $G$ is the monoid of stable self homotopy equivalences of spheres, $A(*)_{1}$ is the one-component of Waldhausen's $A$-theory of a point, and $A^{S}(*)_{1}$ the corresponding space for stable $A$-theory [Wa II]. $A(*)_{1}$ comes equipped with a multiplicative infinite loop space structure, induced from smash product of spaces. As stabilization $A(*)_{1} \rightarrow A^{S}(*)_{1}$ is coherently compatible with smashing, $A^{S}(*)_{1}$ inherits a product from $A(*)_{1}$. By [Wa C] the functor taking finite based sets to based spaces followed by stabilization induces a spectrum level equivalence $S G=Q\left(S^{0}\right)_{1} \rightarrow A(*)_{1} \rightarrow A^{S}(*)_{1}$.

We will define the multiplicative infinite loop space structure on $W h^{\text {Diff }}(*)$ by setting $W h_{\otimes}^{D i f f}(*)$ to be the fiber of the trace map $A(*)_{1} \rightarrow A^{S}(*)_{1} \cong S G$.

We proceed to construct a model for the diagram above using a modified version of Waldhausen's manifold models for the spaces related to $A$-theory [Wa M, $\S 1$ ]. The original manifold models admit a product corresponding to smash product of spaces, up to homotopy, as indicated in [Wa C, p. 399]. It is not clear, however, how to account for higher coherence homotopies in this set-up, nor how to geometrically obtain a spectrum map from $B O$ into $A^{S}(*)_{1}$. This will be transparent in our model.

For simplicity we will only consider manifold models for $A(X)$ when $X=*$ is a point, although similar constructions could be made for $X$ an abelian topological monoid. We shall stably approximate Waldhausen's partitions in $D^{n} \times I$ by partitions in $S^{n} \times I$, and further by codimension zero submanifolds of $S^{n+1}$ which are standardized near infinity. Here $S^{n} \times I$ approximates $S^{n+1}$ by extending the boundary components to the origin and infinity, respectively. Our constructions will then be stably equivalent to the original ones.

Definitions 1.1. Let $\mathbb{R}^{n}$ have the supremum metric $\|\vec{x}\|=\sup _{i}\left|x_{i}\right|$, topologize $S^{n}$ as the one-point compactification of $\mathbb{R}^{n}$ based at infinity, and give $S^{\infty}$ the 
direct limit topology. For a (large) number $\delta$ we call the neighborhood of $* \in S^{\infty}$ consisting of $\vec{x}$ with $\|\vec{x}\| \geq \delta$ a $\delta$-neighborhood of infinity. Let $M^{\prime} \subset S^{n}$ be a compact codimension zero submanifold. For a sequence of positive numbers $\epsilon=\left(\epsilon_{i}\right)$ we call the neighborhood $M$ of $M^{\prime}$ in $S^{\infty}$ consisting of $\vec{x}$ for which there exists a $\vec{y} \in M^{\prime}$ with $\left|x_{i}-y_{i}\right| \leq \epsilon_{i}$ for all $i$ an $\epsilon$-thickening of $M^{\prime}$. The thickenings of compact manifolds in $S^{\infty}$ which contain a standard neighborhood of infinity will be our partitions.

We now follow [Wa M, $\S 1$ ]. Let $\delta \gg 0$ be a fixed number. A partition is a subspace $M \subset S^{\infty}$ which is the union of an $\epsilon$-thickening of some compact codimension zero submanifold $M^{\prime} \subset S^{n}$, for some $\epsilon, M^{\prime}$ and $S^{n}$, and the $\delta$-neighborhood of the base point at infinity. This must be interpreted in a suitable category of manifolds, as explained in [Wa M, $\S 1$ and $\S 6]$. We will work with smooth manifolds with corners, i.e. the $D I F F$ category.

Define $\mathcal{P}=\mathcal{P}(*)$. to be the simplicial set with $k$-simplices the locally trivial families of partitions parametrized over a simplex $\Delta^{k}$. The $\epsilon$ and $M^{\prime}$ may vary through such a family, but there should exist a global (maximal) $n$ for each simplex. $\mathcal{P}$ can be made into a simplicial category with a morphism from $M$ to $N$ if $M \subseteq N$, and we let $h \mathcal{P}$ denote the simplicial subcategory where the morphisms are (roughly) the homotopy equivalences. Precisely the morphisms in $h \mathcal{P}$ are $M \rightarrow N$ such that $M$ and $N$ are thickenings of some $M^{\prime} \subseteq N^{\prime} \subset S^{n}$, with both inclusions

$$
\partial M^{\prime} \subset \longrightarrow N^{\prime}-\operatorname{int}\left(M^{\prime}\right) \longleftrightarrow \partial N^{\prime}
$$

homotopy equivalences. Let $h \mathcal{P}_{k}^{m}$ be the full simplicial subcategory of $h \mathcal{P}$ where $M$ has the homotopy type of a wedge of $k m$-spheres, or more precisely, the component of $h \mathcal{P}$ containing a thickening of an $M^{\prime} \subset S^{n}$ obtained by adding $k$ trivial $m$-handles to a $\delta$-neighborhood of infinity. Let the simplicial set $\mathcal{P}_{k}^{m}$ be given by its objects.

We now discuss stabilization, the additive partial monoid structure on $h \mathcal{P}$, and the coherently associative smash product.

Our thickened manifolds $M \subset S^{\infty}$ are already stabilized with respect to the containing dimension, but we can also stabilize (the homotopy type of) $M$ itself by suspensions. Suppose $M$ is a partition obtained by adding a $\delta$-neighborhood of infinity to an $\epsilon$-thickening of $M^{\prime} \subset S^{n}$. Let $M_{-}^{\prime} \subset \mathbb{R}^{n}$ be $M^{\prime}$ with the point at infinity removed. Then the cartesian product $M_{-}^{\prime} \times \mathbb{R}^{1} \subset \mathbb{R}^{n} \times \mathbb{R}^{1} \cong \mathbb{R}^{n+1}$ is a compact codimension zero submanifold away from a neighborhood of infinity, and the union of its $\epsilon$-thickening and the $\delta$-neighborhood of infinity is the stabilization of $M$. This process may actually better be viewed as smashing with $S^{1}$, as we shall see momentarily.

We can add two partitions if they are disjoint away from the $\delta$-neighborhood of infinity, by forming their union. This provides an additive partial monoid structure on $\mathcal{P}$ (and similarly for $h \mathcal{P}$ ), which we can use to group complete the nerve of $\mathcal{P}$, as the inclusion of composable $k$-tuples of partitions into all $k$-tuples of partitions is stably an equivalence.

To multiply two partitions together, we will use an identification $\mathbb{R}^{\infty} \times \mathbb{R}^{\infty} \cong \mathbb{R}^{\infty}$, say given by shuffling the coordinates: $\left(\left(x_{i}\right),\left(y_{i}\right)\right) \mapsto\left(x_{1}, y_{1}, x_{2}, y_{2}, \ldots\right)$. This pairing satisfies associativity and commutativity up to the usual coherent isomorphisms. Now suppose we are given two partitions $M$ and $N$, which are $\epsilon_{M^{-}}$and 
$\epsilon_{N}$-thickenings of $M^{\prime} \subset S^{m}$ and $N^{\prime} \subset S^{n}$ respectively. We shall define their product $M \otimes N$, which has the homotopy type of $M \wedge N$, by thickening the cartesian product of $M_{-}^{\prime}$ and $N_{-}^{\prime}$ and adding a $\delta$-neighborhood of infinity. Explicitly

$$
M^{\prime} \wedge N^{\prime} \cong\left(M_{-}^{\prime} \times N_{-}^{\prime}\right)+\subset S^{\infty} \wedge S^{\infty}
$$

is a compact subspace of $S^{n} \wedge S^{m} \subset S^{\infty} \wedge S^{\infty} \cong S^{\infty}$, which is a codimension zero submanifold of $S^{n} \wedge S^{m}$ away from infinity. We shuffle the thickening distances $\epsilon_{M}$ and $\epsilon_{N}$ in the obvious way into $\epsilon$, and let $M \otimes N$ be the union of the $\epsilon$-thickening of $M^{\prime} \wedge N^{\prime}$ with the standard $\delta$-neighborhood of infinity.

$n$-fold suspension stabilization may now be realized as forming the $\otimes$-product with a thickening of $S^{n} \subset S^{\infty}$.

We let $\mathcal{P}^{m}=\coprod_{k \geq 0} \mathcal{P}_{k}^{m}$, and denote its nerve as a partial additive monoid by $N_{\Gamma}\left(\mathcal{P}^{m}\right)$. Consider the double square

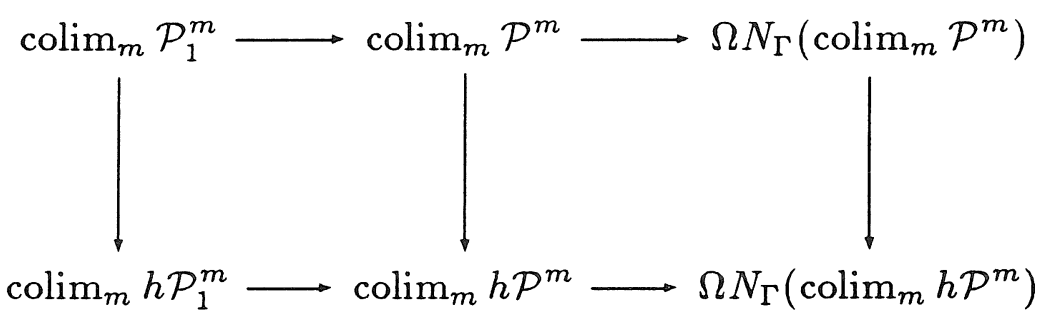

where the direct limits are formed by suspensions. Recall the terminology from [Wa $\mathrm{M}, \S 1$ and $\S 3$ ], where $h \underline{P}_{k}^{m}\left(X \times J^{n}\right)$ is the simplicial category of parametrized partitions of $X \times J^{n} \times I$ obtained by adding $k$ trivial $m$-handles to a standard picture near $X \times J^{n} \times\{0\}$, under homotopy equivalences. The notations obtained by omitting $h, m$ or $k$ are derived analogously to the cases for $\mathcal{P}$ above. The tube space $T^{\text {Diff }}$ is equivalent to $\operatorname{colim}_{m, n} \underline{P}_{1}^{m}\left(D^{m+n}\right)$.

Lemma 1.2. There is a chain of homotopy equivalences linking the outer square above to the homotopy cartesian square :

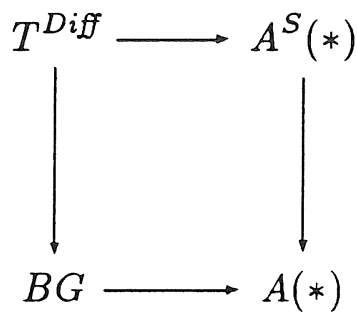

Proof. There are maps

$$
h \mathcal{P}_{k}^{m} \longleftrightarrow h \underline{P}_{k}^{m}\left(S^{n}\right) \longrightarrow h \underline{P}_{k}^{m}\left(D^{n+1}\right)
$$

which become stable equivalences as $n$ tends to infinity. Here we embed Waldhausen's partitions of $S^{n} \times I$ into a partition of $S^{\infty}$ by thinking of $S^{n} \times I$ as an annulus missing the origin and a neighborhood of infinity, and then thickening. See also [Wa C, p. 400] for a discussion of this kind of rewriting. 
These maps are sufficiently natural when $k, m$, and $n$ vary to assemble into the claimed chains of equivalences. The statement that the square is homotopy cartesian is [Wa M, Proposition 3.1].

We have thus recognized the homotopy types in the outer square $(*)$. The (coherently) associative and commutative $\otimes$-product is respected by the maps in that diagram, and gives rise to multiplicative infinite loop space structures on the leftmost spaces, and the one-components of the rightmost spaces. Hence these components of the outer square of $(*)$ form a square of infinite loop spaces.

We can also map $B O$ into $T^{D i f f}$ as a spectrum map, using the additive (Whitney sum) infinite loop space structure on $B O$. To do this, chose a (small) number $\epsilon>0$. Take as a model for $B O(m)$ the Grassmannian space of $m$-planes in $R^{\infty}$, and map a vector space $V$ to its $\epsilon$-thickening in $R^{\infty} \subset S^{\infty}$, with a $\delta$-neighborhood of infinity added. This map takes Whitney sum of vector spaces to the $\otimes$-product of partitions, and is thus compatible with stabilization in $m$. The reason why we use the supremum metric on $\mathbb{R}^{\infty}$ is to make this map commute precisely with these pairings.

This map is clearly homotopic to that of [Wa M, Proposition 3.2]. We have thus proved :

Theorem 1.3. There exists a homotopy commutative square of spectra

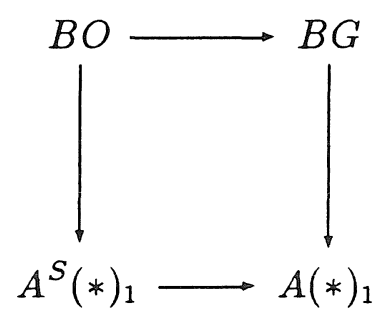

where $B O=B O_{\oplus}$ carries the additive infinite loop space structure from Whitney sum, and $B G, A^{S}(*)_{1} \cong Q\left(S^{0}\right)_{1}=S G$ and $A(*)_{1}$ carry the multiplicative infinite loop space structures from smash product. The map $B O \rightarrow B G$ is the delooped $J$-homomorphism, the map $A^{S}(*)_{1} \rightarrow A(*)_{1}$ is equivalent to the map induced by including the category of finite based sets into the category of finite spaces, and the map $B G \rightarrow A(*)_{1}$ includes self homotopy equivalences of spheres into homotopy equivalences of more general spaces.

Corollary 1.4. There exists a spectrum map $G / O \rightarrow \Omega W h_{\otimes}^{D i f f}(*)$ from the fiber of $B O \rightarrow B G$ to the fiber of $A^{S}(*)_{1} \rightarrow A(*)_{1}$, which on underlying spaces agrees up to homotopy with Waldhausen's map [Wa M, Corollary 3.4].

As Waldhausen remarks, A. Hatcher has constructed a similar space level map, which may be the same as this one. K. Igusa has also given a geometric description of such a map.

\section{§2. BÖKSTEDT'S MODEL FOR ÉTALE $K$-THEORY}

We apply the construction in $\S 1$ to the question of whether Böksted's twocomplete splitting of the space $\Omega J K(\mathbb{Z})$ off from $\Omega K(\mathbb{Z})$ [Bö] can be lifted to the 
spectrum level. This turns out to reduce to the old problem of how the cokernel of $J$ maps into $K(\mathbb{Z})$; see Theorem 2.1 .

The line of argument follows Bökstedt's paper, with modifications to allow for spectrum level constructions. As all of the homotopies needed for Bökstedt's splittings turn out to lift uniquely to the spectrum level, the added information content of a spectrum level splitting compared to a space level splitting may be viewed as lying entirely in $\S 1$ and the hypothesis about the cokernel of $J$.

Let all spectra be completed at the prime two in this section. The spectrum $J K(\mathbb{Z})$ can be defined as the homotopy fiber of the composite map of spectra

$$
k O=\mathbb{Z} \times B O \stackrel{\psi^{3}-1}{\longrightarrow} B \operatorname{Spin} \stackrel{c}{\longrightarrow} B S U
$$

where $\psi^{3}$ is the Adams operation, and $c$ complexification. Its one component $J K(\mathbb{Z})_{1}$ has a multiplicative infinite loop space structure, given as the homotopy fiber of :

$$
B O_{\otimes} \stackrel{\psi^{3} / 1}{\longrightarrow} B \operatorname{Spin}_{\otimes} \stackrel{c}{\longrightarrow} B S U_{\otimes}
$$

It will be clear later that the exponential cannibalistic equivalences $\rho^{3}: B S O_{\oplus} \rightarrow$ $B S O_{\otimes}$ and $J_{\oplus} \rightarrow J_{\otimes}[\mathrm{AP}],[\mathrm{MST}$, Corollary 4.4] induce an equivalence of spectra from the additive zero component $J K(\mathbb{Z})_{0}$ to $J K(\mathbb{Z})_{1}$. Our constructions will relate $J K(\mathbb{Z})_{1}$ to $K(\mathbb{Z})_{1}$ and $A(*)_{1}$ of the previous section, so it is natural for us to focus on the multiplicative model for the spectrum structure.

Let $J_{\oplus}, J_{\otimes}, C_{\oplus}$ and $C_{\otimes}$ be the additive and multiplicative connected image of $J$ and cokernel of $J$ spectra. There is a non-split fibration (see Theorem 3.1)

$$
C_{\otimes} \longrightarrow S G \stackrel{e}{\longrightarrow} J_{\otimes}
$$

and the usual map $S G=Q\left(S^{0}\right)_{1} \rightarrow A(*)_{1} \rightarrow K(\mathbb{Z})_{1}$.

Theorem 2.1. Let all spaces and spectra be completed at the prime 2.

If the composite map $C_{\otimes} \rightarrow S G \rightarrow K(\mathbb{Z})_{1}$ is null homotopic as a map of spectra, then the natural map $K(\mathbb{Z})_{1} \rightarrow J K(\mathbb{Z})_{1}$ is a split surjection in the category of spectra.

If the space of space maps from $C_{\otimes}$ to $K(\mathbb{Z})_{1}$ is contractible, then the natural map $K(\mathbb{Z})_{1} \rightarrow J K(\mathbb{Z})_{1}$ is a split surjection of spaces.

Note that Mitchell [Mit] has proved the space level version of the first hypothesis above, i.e. that the map $S G \rightarrow K(\mathbb{Z})$ of spaces factors through the image of $J$. The corresponding space level conclusion does not immediately follow, however.

The proof of the Theorem will consist of a series of Lemmas. First we need two preparatory results which follow directly from Adams' presentation [Ad, Chapter 6] of the theorems of Madsen, Snaith and Tornehave, generalizing [MST, Theorem B].

Let $\eta$ denote the essential spectrum map $S^{1} \rightarrow S^{0}$, or the induced map $\Sigma X \rightarrow X$ for any spectrum $X$.

Proposition 2.2. There are no essential spectrum maps $B S O \rightarrow K O$ in degrees congruent to $-1,-2,-3$ or -5 modulo 8 . 
All spectrum maps $B S O \rightarrow$ Spin and $B B S O \rightarrow$ Spin factor through $\eta$ and $\eta^{2}$ respectively.

Let $[X, Y]$ denote the homotopy classes of spectrum maps from $X$ to $Y$, when $X$ and $Y$ are spectra.

Proof. The first part is immediate from the universal coefficient theorem [Ad, 6.4.7]

$$
[B S O, K O]_{*} \cong \operatorname{Hom}_{\pi_{*} K O}\left(K O_{*} B S O, \pi_{*} K O\right)
$$

the fact that $K O_{*} B S O$ is a free $\pi_{*} K O$-module on (countably many) generators in degree zero, and the vanishing of $\pi_{*} K O$ in the degrees mentioned.

For the second part, note that left composition with $\eta$ defines a natural transformation of degree one of both sides of the formula above. As multiplication by $\eta$ maps $\pi_{0} K O$ onto $\pi_{1} K O, \eta_{*}:[B S O, K O] \rightarrow[B S O, K O]_{1}$ is onto, and the result follows.

The case of $\eta^{2}$ is entirely similar.

Corollary 2.3. There are no essential spectrum maps $B S O \rightarrow S U$, and any $f: B B S O \rightarrow S U$ with $\pi_{*}(f ; \mathbb{Q})=0$ is null homotopic.

Proof. To see this map $B S O$ or $B B S O$ into the fibration sequence [Mil, §24] or [Ma, Proof of V.5.15] :

$$
B S O \stackrel{\eta}{\longrightarrow} \operatorname{Spin} \stackrel{c}{\longrightarrow} S U \longrightarrow B B S O
$$

Both statements now follow from a little chase using Proposition 2.2, and the fact that a spectrum map $B B S O \rightarrow B B S O$ which is trivial on rational homotopy is null homotopic [Ad, 6.4.2].

We now turn to the proof of Theorem 2.1. Recall the null homotopy of the composite $B S O \rightarrow S G \rightarrow K(\mathbb{Z})_{1}$ :

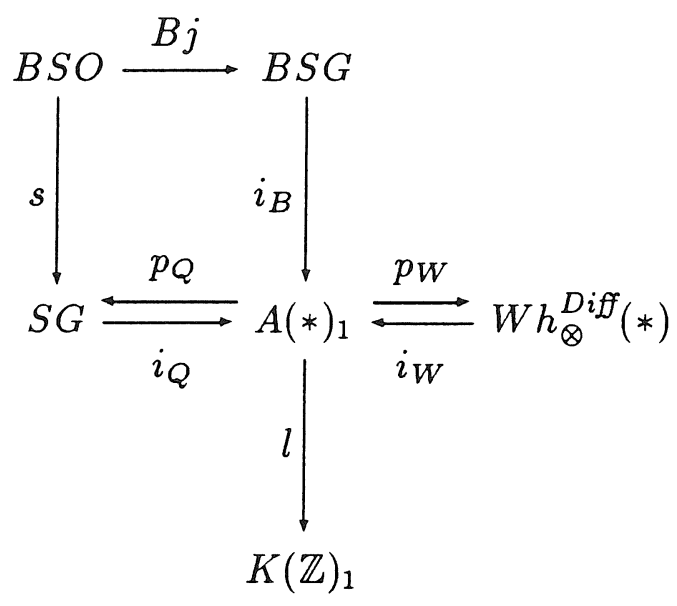

The commuting square is from Theorem 1.3, and the multiplicative splitting of $A(*)_{1}$ into $S G$ and $W h_{\otimes}^{D i f f}(*)$ from [Wa II] and [Wa C]. $l$ denotes the linearization map. 
Lemma 2.4. The composite $l \circ i_{Q} \circ s: B S O \rightarrow S G \rightarrow K(\mathbb{Z})_{1}$ is null homotopic as a map of spectra.

Proof. Bökstedt's argument applies unchanged: $s$ is homotopic to the composite $p_{Q} \circ i_{B} \circ B j$, and $l \circ i_{B}$ is null homotopic. Thus $l \circ i_{Q} \circ s$ is homotopic to $l \circ i_{W} \circ p_{W} \circ i_{B} \circ$ $B j$ (up to sign), which factors through the null map $S G \rightarrow A(*)_{1} \rightarrow W h_{\otimes}^{D i f f}(*)$.

Next we consider the map. s. Bökstedt and Waldhausen proved that the composite $p_{Q} \circ i_{B}: B S G \rightarrow A(*)_{1} \rightarrow S G$ is homotopic to multiplication by $\eta$ as a map of spaces [Bö-Wa]. Presumably this also is true on the spectrum level, and could plausibly be proved by extending the methods of $\S 1$. We will however only need, and prove, the following weaker statements.

Lemma 2.5. The only essential spectrum map $f: B S O_{\otimes} \rightarrow J_{\otimes}$ is the composite $t_{\otimes}=\zeta_{\otimes} \circ \eta$, which is nontrivial on homotopy. Hence spectrum maps $B S O_{\otimes} \rightarrow J_{\otimes}$ are detected on underlying spaces.

Corollary 2.6. The composite e os:BSO $\rightarrow S G \rightarrow J_{\otimes}$ is homotopic as a map of spectra to the composite

$$
B S O \stackrel{B j}{\longrightarrow} B S G \stackrel{\eta}{\longrightarrow} S G \stackrel{e}{\longrightarrow} J_{\otimes}
$$

Proof of Lemma 2.5. Consider the diagram:

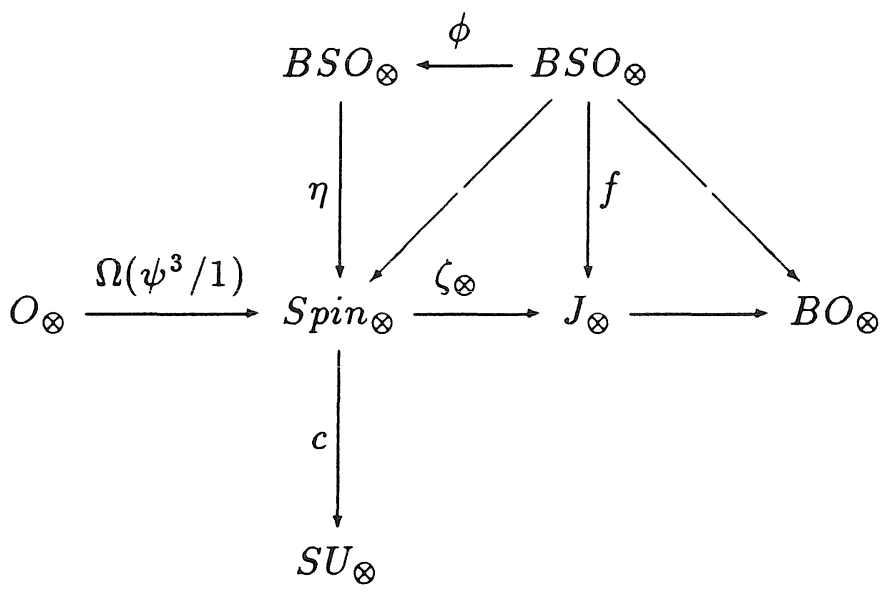

As $f$ is trivial on rational homotopy, and $B O_{\otimes} \cong B O(1) \vee B S O_{\otimes}$, the composite of $f$ into $B O_{\otimes}$ is null homotopic. Hence there exists a lift of $f$ into $S p i n_{\otimes}$, whose composite with $c$ to $S U_{\otimes}$ is null by Corollary 2.3. Thus there exist a lifting $\phi$, which (in additive notation) can be written as a series

$$
\phi \cong \epsilon(\phi)+\sum_{r, i} a_{r, i}\left(\psi^{2^{r}+i}-\psi^{i}\right)
$$

by [MST, Lemmas 2.2 and 2.11]. Here $a_{r, i}$ is only nonzero for certain odd $i$. Each of the terms on the right factor through $\psi^{3}-1$, and as $\psi^{3}-1$ commutes with $\eta$, they contribute nothing when composed with $\zeta_{\otimes}\left[R_{0}\right]$. Thus we may make use of 
the choice in selecting a lifting $\phi$ of $f$, and can indeed take $\phi$ to be a scalar multiple $\epsilon(\phi)$ of the identity. The Lemma follows, as $\eta$ and thus $t_{\otimes}$ has order two.

Recall from [Bö] the diagram of fibration sequences in its multiplicative version :

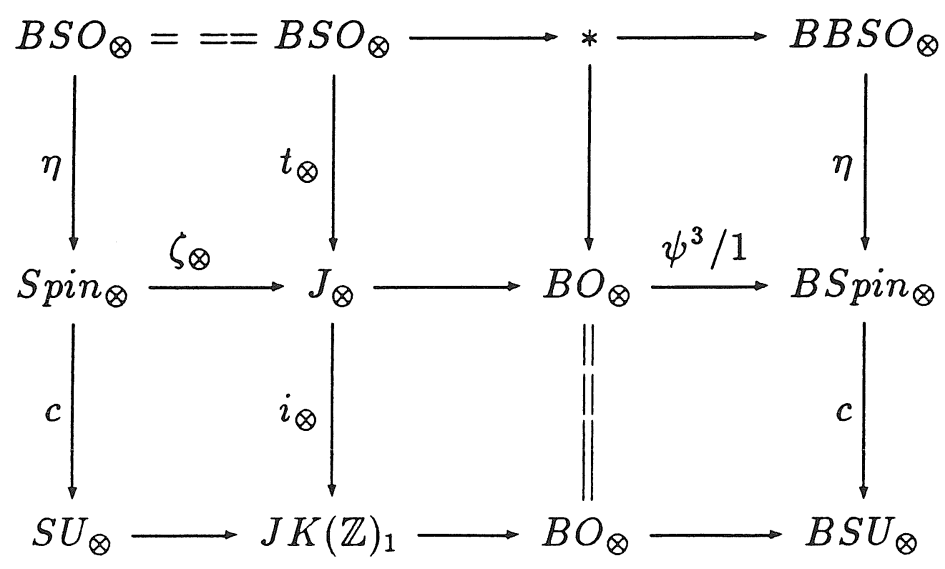

Lemma 2.7. The composites $t_{\otimes} \circ \rho^{3}$ and $e \circ s$ are homotopic as maps of spectra.

Proof. We use

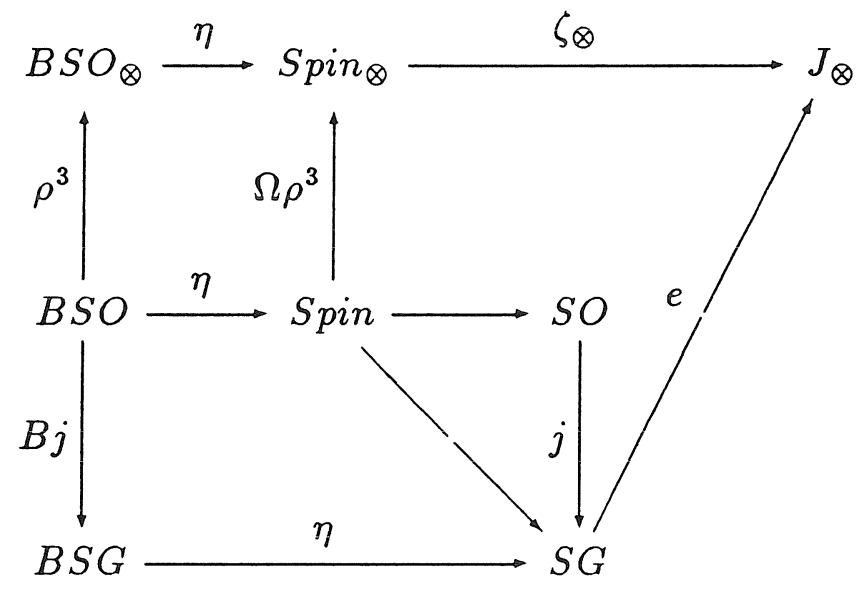

which commutes by naturality of multiplication by $\eta$ and the $J$-theory diagram of [Ma, p. 107] :

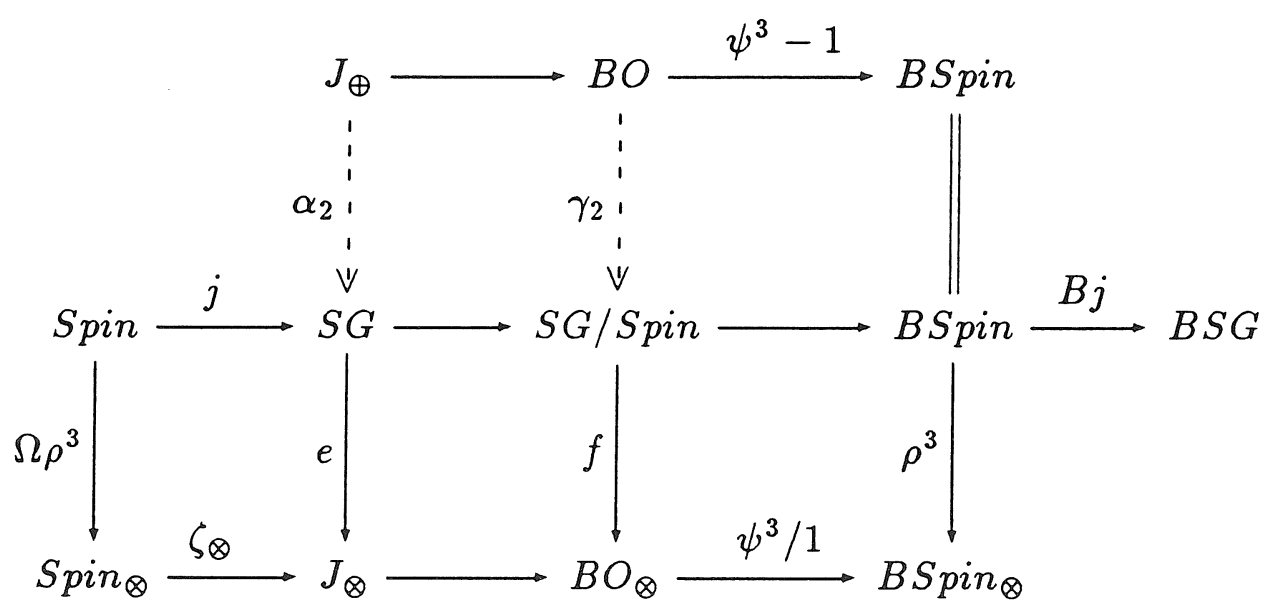


where $\alpha_{2}$ and $\gamma_{2}$ are not $H$-maps, but the composites $e \circ \alpha_{2}$ and $f \circ \gamma_{2}$ can be chosen as spectrum maps. The bottom left square can be recognized in the diagram above.

We proceed to try to produce a map from $J K(\mathbb{Z})_{1}$ to $K(\mathbb{Z})_{1}$, not by using the non-deloopable splitting $\alpha_{2}$, but by lifting the map from the (spectrum level) cofiber $g: B \mathrm{fib}(s) \rightarrow K(\mathbb{Z})_{1}$ over $B \mathrm{fib}(s) \rightarrow J K(\mathbb{Z})_{1}$. Diagrammatically,

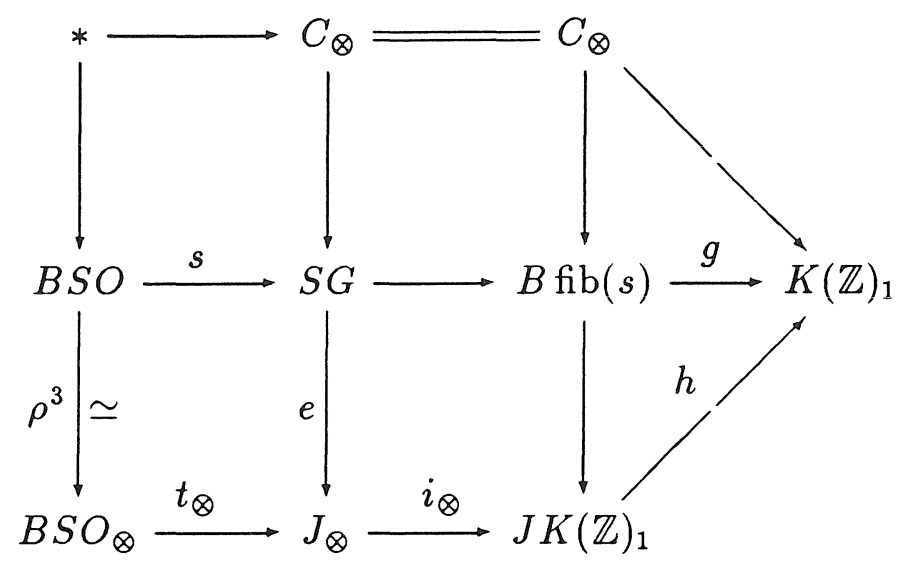

the spectrum map $h: J K(\mathbb{Z})_{1} \rightarrow K(\mathbb{Z})_{1}$ exists as a lifting if and only if the composite $C_{\otimes} \rightarrow B \mathrm{fib}(s) \rightarrow K(\mathbb{Z})_{1}$ is null homotopic. This is the same map as in the hypothesis of Theorem 2.1. The left part of the diagram is a square of fibrations, and the map $g: B \mathrm{fib}(s) \rightarrow K(\mathbb{Z})_{1}$ is given by the null homotopy of Lemma 2.4.

We note that Mitchell's result that $C_{\otimes} \rightarrow K(\mathbb{Z})_{1}$ is null homotopic on the space level only suffices to define a map from the mapping cone (cofiber) of $C_{\otimes} \rightarrow B$ fib $(s)$ to $K(\mathbb{Z})_{1}$, not from the spectrum level cofiber $J K(\mathbb{Z})_{1}$.

However, by "Ganea theory" [Gan] there exists a tower of obstructions to this space level lifting extension problem, lying in the group of homotopy classes of space level maps $C_{\otimes} * \Omega J K(\mathbb{Z})_{1} * \cdots * \Omega J K(\mathbb{Z})_{1}$ to $K(\mathbb{Z})_{1}$. Here $*$ denotes the join of spaces, and there are one or more factors $\Omega J K(\mathbb{Z})_{1}$. To see this, consider the tower of spaces

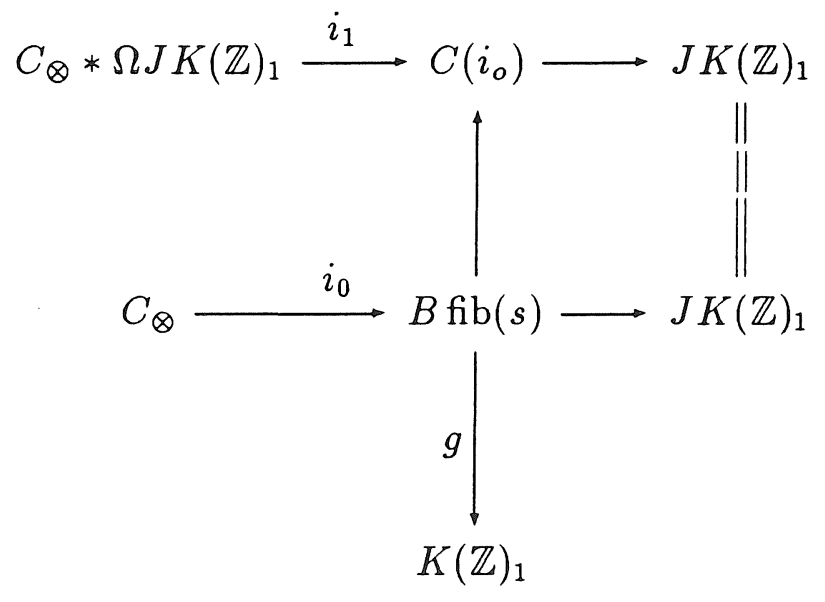


where the rows are fibrations and $C\left(i_{s}\right)$ the mapping cone on $i_{s}$. The map $g$ extends to $J K(\mathbb{Z})_{1}$ precisely if all the maps from the fibers to $K(\mathbb{Z})_{1}$ are null homotopic. This proves the second part of Theorem 2.1, as in [Bö].

It appears unlikely that Mitchell's argument will extend to resolve these higher obstruction questions. Let us therefore make the following assumption :

Hypothesis 2.8. The composite spectrum map $C_{\otimes} \rightarrow S G \rightarrow K(\mathbb{Z})_{1}$ is null homotopic.

Granted this, a lifting $h: J K(\mathbb{Z})_{1} \rightarrow K(\mathbb{Z})_{1}$ of $g$ exists. Bökstedt defines a ring spectrum map $\Phi: K(\mathbb{Z}) \rightarrow J K(\mathbb{Z})$. In fact there exists a commutative square of rings

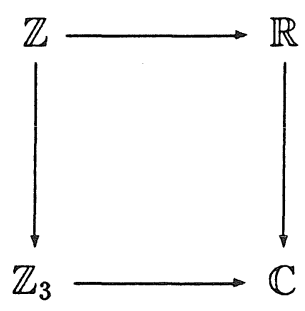

where $\mathbb{Z}_{3}$ denotes the 3 -adic integers, and an equivalence $K\left(\mathbb{Z}_{3}\right) \rightarrow K\left(\mathbb{F}_{3}\right)$ induced by the residue field homomorphism [Gab], which composes with a Brauer lift $K\left(\mathbb{F}_{3}\right) \rightarrow K(\mathbb{C})$ to give the map on $K$-theory induced by the lower horizontal arrow. $J K(\mathbb{Z})$ is a cover of the pullback $P B$ of the induced square

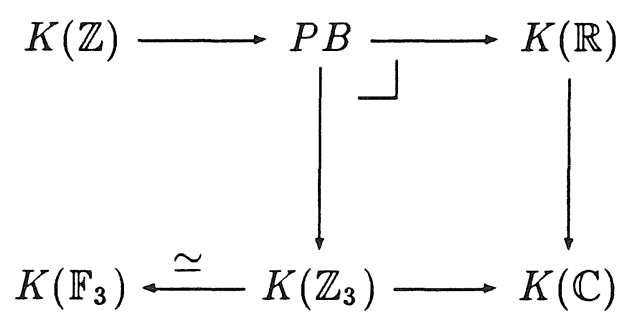

and $\Phi: K(\mathbb{Z}) \rightarrow J K(\mathbb{Z})$ is a lift of the $\operatorname{map} K(\mathbb{Z}) \rightarrow P B$.

Lemma 2.9. There is a homotopy commutative diagram of spectra :

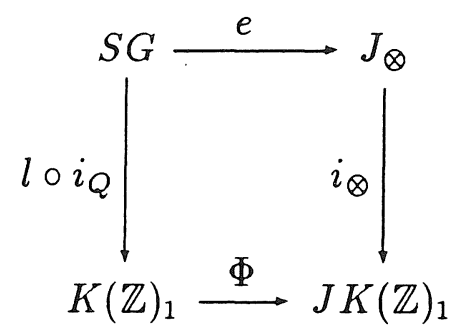

Presumably there is a similar result for the additive spectra.

Proof. As in [Bö, Proof of 2.1] we have a covering $\mathbb{Z} \rightarrow J K(\mathbb{Z})_{1} \rightarrow P B_{1}$, and as there are no homomorphisms $\pi_{1}(S G) \cong \mathbb{Z} / 2 \rightarrow \mathbb{Z}$ it suffices to compare maps into $P B_{1}$. This in turn reduces to comparing maps into $B O_{\otimes}$ and $K\left(\mathbb{Z}_{3}\right)_{1} \cong K\left(\mathbb{F}_{3}\right)_{1}$, as well as the homotopy linking the composites into $B U_{\otimes}$.

Both composites $S G \rightarrow K\left(\mathbb{F}_{3}\right)_{1}$ agree with the map given by taking a finite set to the $\mathbb{F}_{3}$-vector space it generates, as is seen by the "discrete model" for $J_{\otimes}$ described e.g. in [Ma, VIII.3.1]. 
The composites $S G \rightarrow B O_{\otimes}$ are homotopic on the underlying space level by Bökstedt's argument, and thus on the spectrum level by [Li] or [Ma, V.7.9].

It remains to compare the homotopies of maps into $B U_{\otimes}$, or equivalently a lifting $S G \rightarrow U_{\otimes}$. Now there is only one such, the null map, which follows from the $K$-theoretic equivalence $S G \rightarrow J_{\otimes}$ [HS] and the Lemma below.

Lemma 2.10. There are no essential spectrum maps $f: J_{\otimes} \rightarrow U_{\otimes}$.

Proof. Consider maps of the fibration sequence below to $U_{\otimes}$ :

$$
\operatorname{Spin}_{\otimes} \stackrel{\zeta_{\otimes}}{\longrightarrow} J_{\otimes} \longrightarrow B O_{\otimes}
$$

$J_{\otimes}$ is rationally a point, so $f \circ \zeta_{\otimes}$ is null on rational homotopy. By the second part of Corollary 2.3 (desuspended two degrees) it follows that $f$ extends over $J_{\otimes} \rightarrow B O_{\otimes}$. The extension $B O_{\otimes} \rightarrow U_{\otimes}$ is trivial, because of the first part of Corollary 2.3 and the absence of maps $B O(1) \rightarrow U_{\otimes}[\mathrm{AH}]$ or $B S O \rightarrow U(1)$.

We may now prove Theorem 2.1. Again adapting Bökstedt's proof, we consider the diagram of vertical fibrations

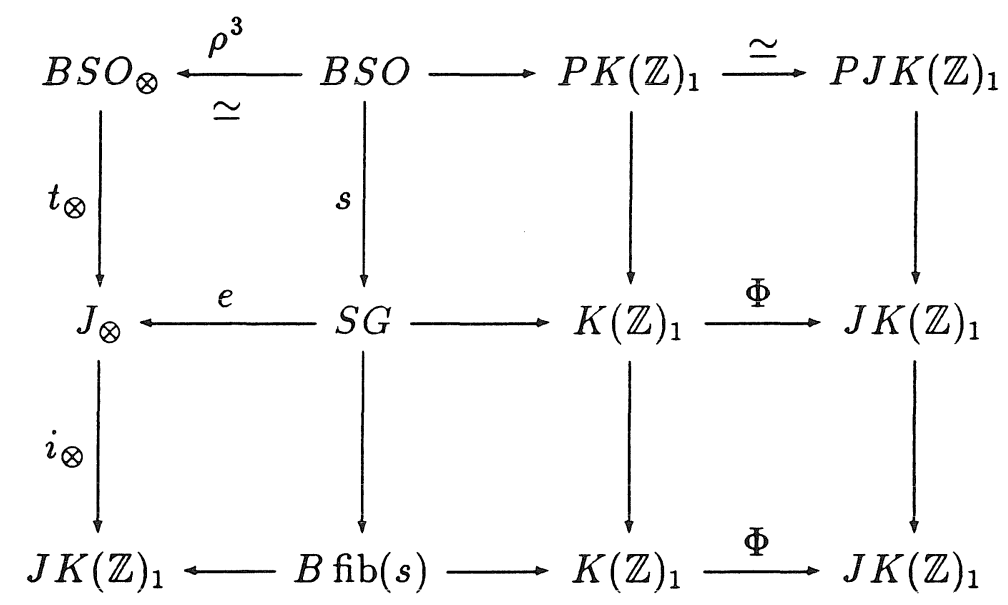

where there exists unique lifting maps from the left to the right by the $K$-acyclicity of $C_{\otimes}=f i b(e)$ and $K$-locality of $J K(\mathbb{Z})_{1}$ (or rather, that $J K(\mathbb{Z})_{1}$ is the connected cover of its $K$-localization). If we assume Hypothesis 2.8 the bottom lifting map may be taken to be $\Phi \circ h$. By Lemma 2.9 the middle lifting map is homotopic to $i_{\otimes}: J_{\otimes} \rightarrow J K(\mathbb{Z})_{1}$, and the three horizontal fibers form a fibration

$$
B S O_{\otimes} \stackrel{a}{\longrightarrow} B S O_{\otimes} \longrightarrow \mathrm{fib}(\Phi \circ h)
$$

where $t_{\otimes} \circ a \simeq t_{\otimes}$. By [Bö, Lemma 1.10], the map $a$ is a homotopy equivalence of spaces, and thus of spectra. Hence $f i b(\Phi \circ h)$ is contractible and $\Phi$ is a split surjection. This completes the proof of Theorem 2.1 .

Remark 2.11. Bökstedt, Hsiang and Madsen [BHM] have constructed a cyclotomic trace map trc: $K(\mathbb{Z}) \rightarrow T C(\mathbb{Z}, p)$ for each prime $p$. Suppose, as follows for $p$ odd from Hypothesis 4.15 in $[\mathrm{BM}]$, that $T C(\mathbb{Z}, p)$ is the connected cover of its $K$ localization. Then the analog of Hypothesis 2.8 would be satisfied for maps into 
$T C(\mathbb{Z}, p)$, and the constructions above may provide a factorization of trc through $\Phi: K(\mathbb{Z})_{1} \rightarrow J K(\mathbb{Z})_{1}$. Such a factorization would exist by $\mathrm{R}$. Thomason's theorem $[\mathrm{Th}]$ and the $K$-locality Hypothesis cited.

Remark 2.12. The composite $\Phi \circ l: A(*)_{1} \rightarrow K(\mathbb{Z})_{1} \rightarrow J K(\mathbb{Z})_{1}$ induces a map $\Omega W h_{\otimes}^{D i f f}(*) \rightarrow G / O_{\otimes}$ on the fibers of the natural maps from $S G$ into $A(*)_{1}$ or $J K(\mathbb{Z})_{1}$ respectively. Here $G / O_{\otimes} \simeq B S O_{\otimes} \vee C_{\otimes}$ is defined as the pullback in a square

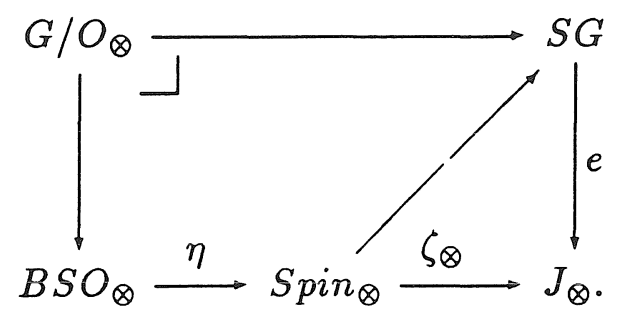

The composite $G / O \rightarrow \Omega W h_{\otimes}^{D i f f}(*) \rightarrow G / O_{\otimes}$ is a rational equivalence, but fails to be an equivalence on the $K$-acyclic part, as is seen by mapping $C_{\otimes}$ into this sequence.

\section{§3. TWice a sPlitting MaP}

J. Tornehave proved the following result, following a discussion with M. Bökstedt where the author played a minor part.

Theorem 3.1. (Tornehave) The cofiber map $J_{\otimes} \rightarrow B C_{\otimes}$ of $e: S G \rightarrow J_{\otimes}$ has order precisely two as a spectrum map.

Proof. At odd primes, the map is null homotopic. Localized at two we have the diagram of spectra

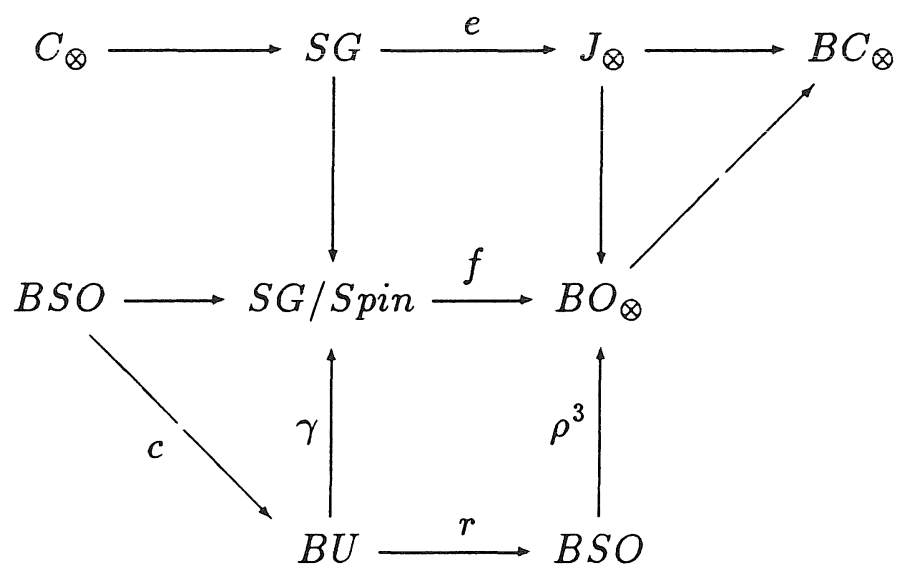

where $\gamma$ is E. Friedlander's spectrum level lifting of a solution to the Adams conjecture to $B U$ [Fr, Theorem 9.2]. The composite $r \circ c: B S O \rightarrow B S O$ is homotopic to twice the identity, and the composite $S G / \operatorname{Spin} \rightarrow B O_{\otimes} \rightarrow B C_{\otimes}$ is null homotopic. Thus $B S O \rightarrow B C_{\otimes}$ has order two. $\rho^{3}$ maps as an equivalence to $B S O_{\otimes}$, so $B S O_{\otimes} \rightarrow B C_{\otimes}$ has order two, and it remains to prove the same for the factor $B O(1) \rightarrow B O_{\otimes} \rightarrow B C_{\otimes}$. But this is obvious, as the identity map of $B O(1)$ has order two. Consequently $J_{\otimes} \rightarrow B O_{\otimes} \rightarrow B C_{\otimes}$ also has order two. 
Remark 3.2. Thus twice the identity map of $J_{\otimes}$ factors over $e$, and if a suitable map $B S O_{\otimes} \rightarrow C_{\otimes}$ vanishes, we obtain a spectrum map $J K(\mathbb{Z})_{1} \rightarrow B$ fib $(s)$. Composing with $B$ fib $(s) \rightarrow K(\mathbb{Z})_{1}$ yields a spectrum map $J K(\mathbb{Z})_{1} \rightarrow K(\mathbb{Z})_{1}$ which would be homotopic to twice the hypothetical map $h$, at least on looped underlying spaces. This indicates that it should be possible to find a map $2 \cdot h$ such that the composite

$$
J K(\mathbb{Z})_{1} \stackrel{2 \cdot h}{\longrightarrow} K(\mathbb{Z})_{1} \stackrel{\Phi}{\longrightarrow} J K(\mathbb{Z})_{1}
$$

is homotopic to twice the identity map.

In general, as a tool for proving that a pair of tentative splitting maps actually provide a spectrum level splitting of $J K(\mathbb{Z})_{1}$ off from $K(\mathbb{Z})_{1}$ (or any other spectrum), we provide the following characterization of some self maps of $J K(\mathbb{Z})_{1}$. Let us again assume that all spectra are completed at the prime two.

Let $v: J_{\otimes} \rightarrow B B S O_{\otimes}$ be the composite

$$
J_{\otimes} \longrightarrow B O_{\otimes} \rightleftarrows B O(1) \longrightarrow B B S O \stackrel{B \rho^{3}}{\simeq} B B S O_{\otimes}
$$

where $B O(1) \rightarrow B B S O$ is the unique essential map, which is null homotopic on underlying spaces. It is the cofiber map extending the nonsplit extension $B S O \rightarrow$ $B O \rightarrow B O(1)$. We shall see in Lemma 3.4 that $v$ is the unique essential map $J_{\otimes} \rightarrow$ $B B S O_{\otimes}$, and in Lemma 3.5 that it has a unique rationally trivial lift $w: J K(\mathbb{Z})_{1} \rightarrow$ $J K(\mathbb{Z})_{1}$.

Proposition 3.3. Let $f: J K(\mathbb{Z})_{1} \rightarrow J K(\mathbb{Z})_{1}$ be a spectrum map with $\pi_{*}(f)=0$. Then $f \simeq *$ or $f \simeq w$.

Lemma 3.4. $\left[J_{\otimes}, B B S O_{\otimes}\right] \cong \mathbb{Z} / 2$ is generated by the map $v$ defined above, with $\Omega^{\infty} v \simeq *$.

Proof. Consider maps of the sequence

$$
\operatorname{Spin}_{\otimes} \stackrel{\zeta_{\otimes}}{\longrightarrow} J_{\otimes} \longrightarrow B O_{\otimes} \longrightarrow B \operatorname{Spin}_{\otimes}
$$

into $B B S O_{\otimes}$. By Proposition 2.2 there are no essential maps $S$ pin $\otimes \rightarrow B B S O_{\otimes}$ or $B S p i n_{\otimes} \rightarrow B B S O_{\otimes}$, so we need only consider maps from $B O_{\otimes} \cong B O(1) \vee B S O_{\otimes}$. Again there are no maps $B S O_{\otimes} \rightarrow B B S O_{\otimes}$, and by [Ma, V.7.7] the only map $B O(1) \rightarrow B B S O \simeq B B S O_{\otimes}$ is that described above.

Lemma 3.5. The spectrum map $v$ has a unique factorization

$$
J_{\otimes} \stackrel{i_{\otimes}}{\longrightarrow} J K(\mathbb{Z})_{1} \stackrel{w}{\longrightarrow} J K(\mathbb{Z})_{1} \longrightarrow B B S O_{\otimes}
$$

through a rationally trivial map $w$. 
Proof. Consider the diagram:

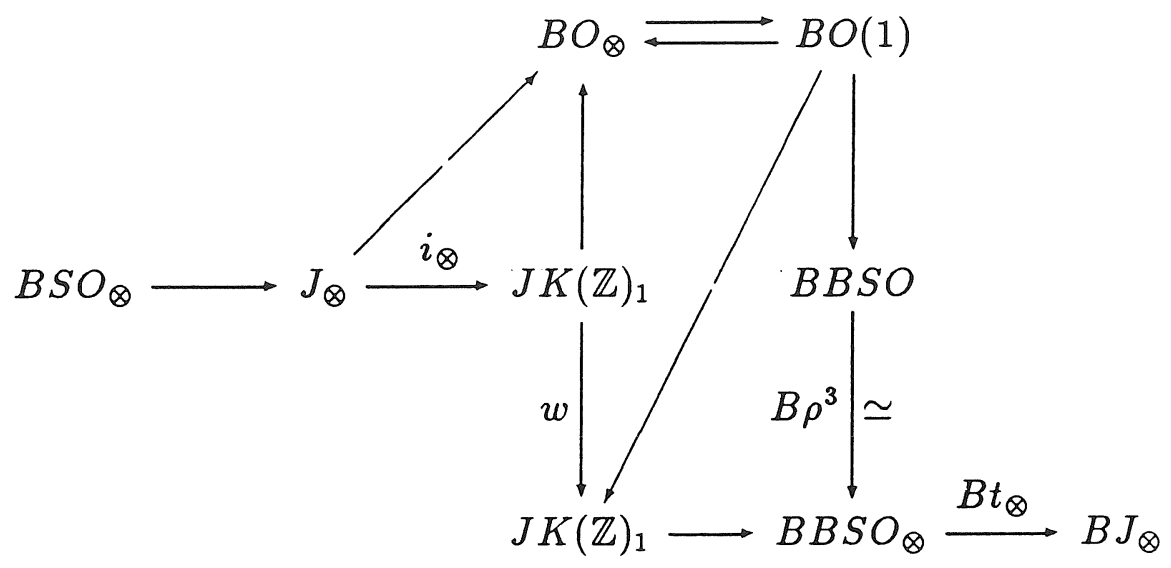

Clearly $v$ extends uniquely through $i_{\otimes}$ to a rationally trivial map $J K(\mathbb{Z})_{1} \rightarrow$ $B B S O_{\otimes}$, as the choices involved differ by rationally detected self maps of $B B S O_{\otimes}$.

The composite $B O(1) \rightarrow B B S O_{\otimes} \rightarrow B J_{\otimes}$ factors through $B \zeta_{\otimes}: B S p i n_{\otimes} \rightarrow$ $B J_{\otimes}$, and the only essential map $B O(1) \rightarrow B S p i n_{\otimes}$ is given as [Ma, V.7.7]

$$
B O(1) \longrightarrow B O_{\otimes} \stackrel{\psi^{3} / 1}{\longrightarrow} B S p i n_{\otimes}
$$

and thus dies when composed with $\zeta_{\otimes}$. Hence there exists a lift $B O(1) \rightarrow J K(\mathbb{Z})_{1}$ as indicated above, and this lift is unique as

$$
0 \longrightarrow\left[B O(1), J_{\otimes}\right] \longrightarrow\left[B O(1), B O_{\otimes}\right] \stackrel{\cong}{\longrightarrow}\left[B O(1), B S p i n_{\otimes}\right]
$$

is exact.

Proof of Proposition 3.3. By Lemmas 3.4 and 3.5 and the rational triviality of $f$, $f$ or $f+w$ fits into a commutative ladder

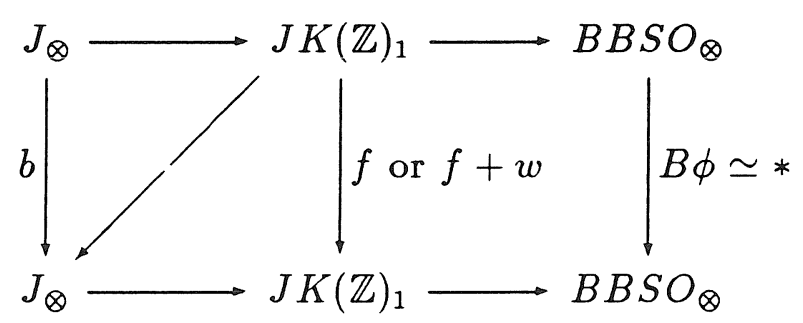

where the (delooped) operation $B \phi$ is rationally trivial and thus null homotopic.

If we now use that $\pi_{*}(f)=0$ it follows that $\pi_{*}(b)$ is null except on certain order two classes $\alpha_{4 k+1}$ in $\pi_{8 k+1}$ which may map to certain other classes $\bar{\alpha}_{4 k+1}$ [Ra, p. 40].

As $\left[S p i n_{\otimes}, B O_{\otimes}\right]=*, b$ also fits into a commutative ladder :

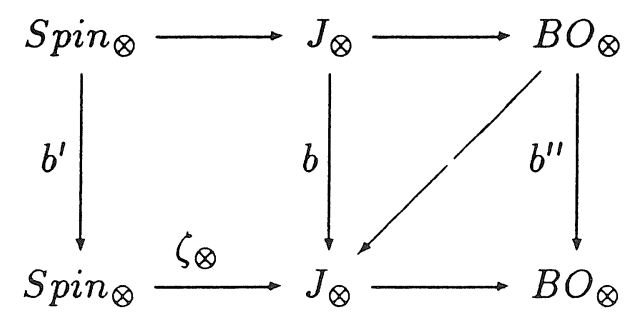


From $\left[S p i n_{\otimes}, S p i n_{\otimes}\right] \cong\left[S p i n_{\otimes}, S O_{\otimes}\right] \cong\left[S O_{\otimes}, S O_{\otimes}\right]$ we see that every choice of $b^{\prime}$ comes from a (looped) operation $\Omega \phi: S O_{\otimes} \rightarrow S O_{\otimes}$. As in the proof of Lemma 2.5 we can write $\phi$ as a series of terms which factor through $\psi^{3} / 1: B O_{\otimes} \rightarrow B S p i n_{\otimes}$, and whose left composite with $\zeta_{\otimes}$ vanishes, together with a scalar multiple of the identity. We may thus choose $b^{\prime}$ to be such a scalar multiple of the identity, and by the near vanishing of $\pi_{*}(b), b^{\prime} \simeq *$.

Thus the self map $b^{\prime \prime}: B O_{\otimes} \rightarrow B O_{\otimes}$ above factors through $J_{\otimes}$ which is rationally trivial, and is thus null homotopic. Hence $b$ factors

$$
J_{\otimes} \longrightarrow B O_{\otimes} \longrightarrow \operatorname{Spin}_{\otimes} \stackrel{\zeta_{\otimes}}{\longrightarrow} J_{\otimes}
$$

From $\left[B O(1), \operatorname{Spin}_{\otimes}\right]=*$, Proposition 2.2 , and the null homotopy $\zeta_{\otimes} \circ\left(\psi^{3} / 1\right) \simeq *$ again, the map $B O_{\otimes} \rightarrow S p i n_{\otimes}$ may be taken to be a scalar multiple of $\eta$.

Thus $b$ factors

$$
J_{\otimes} \longrightarrow J K(\mathbb{Z})_{1} \longrightarrow B O_{\otimes} \stackrel{0 \text { or } \eta}{\longrightarrow} \operatorname{Spin}_{\otimes} \stackrel{\zeta_{\otimes}}{\longrightarrow} J_{\otimes}
$$

and $f$ or $f+w$ factors

$$
J K(\mathbb{Z})_{1} \longrightarrow B O_{\otimes} \stackrel{0 \text { or } \eta}{\longrightarrow} \operatorname{Spin}_{\otimes} \stackrel{\zeta_{\otimes}}{\longrightarrow} J_{\otimes} \stackrel{i_{\otimes}}{\longrightarrow} J K(\mathbb{Z})_{1}
$$

which is null homotopic, as $i_{\otimes} \circ t_{\otimes} \simeq *$.

\section{REFERENCES}

[Ad] J. F. Adams, Infinite loop spaces, Ann. of Math. Studies, vol. 90, Princeton University Press, 1978.

[AP] J. F. Adams and S. B. Priddy, Uniqueness of BSO, Math. Proc. Camb. Philos. Soc. 80 (1976), 475-509.

[AH] D. W. Anderson and L. Hodgkin, The K-theory of Eilenberg-MacLane complexes, Topology 7 (1968), 317-329.

[Bö] M. Bökstedt, The rational homotopy type of $\Omega W h^{D i f f}(*)$, Algebraic Topology, Aarhus 1982, Proceedings (I. Madsen and B. Oliver, eds.), Lecture Notes in Math., vol. 1051, Springer, 1984, pp. 25-37.

[BHM] M. Bökstedt, W. C. Hsiang and I. Madsen, The cyclotomic trace and algebraic $K$-theory of spaces, Århus University preprint (1989).

[BM] M. Bökstedt and I. Madsen, The cyclotomic trace invariant of $\mathbb{Z}_{p}$, in preparation.

[Bö-Wa] M. Bökstedt and F. Waldhausen, The map $B S G \rightarrow A(*) \rightarrow Q\left(S^{0}\right)$, Algebraic Topology and Algebraic $K$-Theory (W. Browder, ed.), Ann. of Math. Studies, vol. 113, Princeton University Press, 1987, pp. 418-431.

[Fr] E. M. Friedlander, Étale homotopy of simplicial schemes, Ann. of Math. Studies, vol. 104, Princeton University Press, 1982.

[Gab] O. Gabber, $K$-theory of Henselian local rings and Henselian pairs, Algebraic $K$-Theory, Commutative Algebra, and Algebraic Geometry, Contemporary Math., vol. 126, AMS, 1992.

[Gan] T. Ganea, A generalization of the homology and homotopy suspension, Comment. Math. Helv. 39 (1965), 295-322.

[HS] L. Hodkin and V. Snaith, The $K$-theory of some more well-known spaces, Illinois J. Math 22 no. 2 (1978), 270-278.

[Li] H. J. Ligaard, Infinite loop maps from $S F$ to $B O_{\otimes}$ at the prime 2, Illinois Journal of Math. 21 (1977), 830-835. 
[MST] I. Madsen, V. Snaith and J. Tornehave, Infinite loop maps in geometric topology, Math. Proc. Camb. Phil. Soc. 81 (1977), 399-429.

[Ma] J. P. May et al., $E_{\infty}$ ring spaces and $E_{\infty}$ ring spectra, Lecture Notes in Math., vol. 577, Springer, 1977.

[Mil] J. Milnor, Morse theory, Ann. of Math. Studies, vol. 51, Princeton University Press, 1963.

[Mit] S. Mitchell, The Morava $K$-theory of algebraic $K$-theory spectra, K-Theory 3 no. 6 (1990), 607-626.

[Ra] D. C. Ravenel, Complex cobordism and stable homotopy groups of spheres, Pure and Applied Math., Academic Press, 1986.

[Ro] J. Rognes, Characterizing BU by homotopy groups, (preprint).

[Th] R. W. Thomason, The Lichtenbaum-Quillen conjecture for $K / \ell_{*}\left[\beta^{-1}\right]$, Current trends in algebraic topology, Part 1 (London, Ont., 1981), CMS Conf. Proc., vol. 2, Amer. Math. Soc., 1982, pp. 117-139.

[Wa II] F. Waldhausen, Algebraic K-theory of topological spaces. II, Algebraic Topology, Aarhus 1978 (Proc. Sympos., Univ. Aarhus, Aarhus, 1978), Lecture Notes in Math., vol. 763, Springer, 1979, pp. 356-394.

[Wa C] F. Waldhausen, Algebraic K-theory of spaces, concordance, and stable homotopy theory, Algebraic Topology and Algebraic K-Theory (W. Browder, ed.), Ann. of Math. Studies, vol. 113, Princeton University Press, 1987, pp. 392-417.

[Wa M] F. Waldhausen, Algebraic K-theory of spaces, a manifold approach, Current trends in algebraic topology, Part 1 (London, Ont., 1981), CMS Conf. Proc., vol. 2, Amer. Math. Soc., 1982, pp. 141-184.

Matematisk Institut, ARhus Universitet and Matematisk Institutt, Universitetet I OSLO 